\title{
An Intelligent Emotion Device Design Based on the Way of Relieving Psychological Pressure for Chinese Internet Employees
}

\author{
Yang $Q i^{1 *}$ \\ ${ }^{1}$ Wuhan University, Wuhan, Hubei province, China
}

\begin{abstract}
With the rapid development of Internet companies, a large amount of capital has been continuously invested into this field and the companies have entered a new stage of development. However, the psychological pressure on their employees has continued to increase and various extreme events have emerged one after another, arousing heated social debates. Based on the background, it is worthy to discuss and research how to help Internet workers relieve their psychological pressure and benefit from humanistic practices in business. To explore novel and effective ways to relieve stress for these employees, intelligent interactive devices combine the healing design and feature multi-sensory integration, so as to promote the efficiency of employees and the long-term development of Internet companies.
\end{abstract}

\section{Introduction}

The market economy can never prosper without efficiency which is the key to ensuring the speed of information circulation. The development of the Internet has transformed the way that people communicate from face-to-face conversation and letter writing to a huge virtual space. As a communication medium, internet not only enables common people to connect with the world, but also meets the companies' pursuit of high efficiency and fast speed. With the advent of the $5 \mathrm{G}$ era, massive amounts of data enable instant interconnection among companies, people and things. In such era, thanks to the rapid development of Chinese Internet companies, China's economy has witnessed ongoing leap-forward growth, with the number of Internet employees up to 16.77 million. Nevertheless, overwork is so widespread in the Internet industry that the relevant discussions have been sparked such as those on "sudden death", "996" and "007" work models, thereby putting growing work pressure on internet workers. Driven by such highintensity work, the employees are in urgent need to find a suitable way to solve their psychological problems and release pressure. That is also the reason why the research adopts the healing concept to design a simple and stressrelieving intelligent interactive device for Internet employees.

\section{Current situation of psychological stress of Internet employees}

\subsection{Sources of psychological stress for Internet employees}

The growth track of Internet companies is different from other traditional counterparts. In the early stage, Internet companies need to vigorously expand user base through free marketing strategies and offline promotion activities, etc. To enhance their attractiveness to potential users, they apply marketing strategies, such as rebates and coupons. Meanwhile, the essence of enhancing customer loyalty is to rapidly upgrade products and continuously optimize users' experience. Then, Internet companies need to invest massive amounts of capital into the stage of rapid growth, a critical period to determine whether they can spring up as an industrial oligarch. Needless to say, each stage requires strong support of human capital. Therefore, in order to attract a large number of highquality talents to join, Internet companies provide attractive incentive policies for employees, including promoting efficiency-wage, which means their salary offer leads the industry. Under such salary package, the companies require employees to maintain high productivity and excellent capability of execution, innovation as well as research and development. In addition, they are also asked to keep sensitive to new technologies. In order to meet such requirements, Internet companies formulate personal KPIs, "996" and "007" work models for employees. In consequence, it is prevailing to see high turnover rate of employees in the 
Internet industry. Furthermore, since non-physical jobs are gradually disenchanted by the public, even this way of working beyond the physical and psychological capacity of ordinary people, still appeals a large number of young people to devote to the Internet industry. Behind material abundance lies the imbalance of selfworth. According to the results of the "Survey on 996 Attitude" by Boss Recruitment, though "996" work schedule is being heavily criticized, $60.6 \%$ of people will still choose it for a monthly salary of 30,000 RMB.

\subsection{The impact of long-term high pressure on Internet employees}

For the above-mentioned factors, long-term overwork has put Internet employees under tremendous psychological pressure, and even many have to face warning signs of health problems. In terms of 2019 Shanghai White-collar Health Index Report, the abnormal rate of Shanghai white-collar workers' physical examinations in 2018 already reached up to $98.75 \%$. Symptoms such as hair loss, insomnia, and depression are not uncommon for Internet workers. "Exchanging life for money" seems to have become a new unhealthy trend in Chinese society, and has been greatly criticized by social scholars as well.

Except for the high-intensity work in Internet companies, China has no shortage of jobs and work styles featuring both efficiency and happiness. However, Internet companies are still the favored by many young people, perhaps driven by the hardworking atmosphere of Chinese society, or influenced by personal desires. Nevertheless, undeniably, if a person has been burdened with such a high-pressure and high-intensity work for a long time, happiness in life seems rare to see for him or her. In the fast-moving society, the values of emotion and happiness for employees receive little attention. Even though risking employees' health and sacrificing their happiness at work can bring about a great boost for company development, behind the flourishing growth of Internet emerge negative emotions of employees like depression.

From a long-time perspective, such work models as "996 (ICU)", "007' and "11116" will gradually erode young people's faith and love for careers. Not only is their personal development limited, but also their hopes for the future will be destroyed little by little, thus further triggering negative emotions at work in society. Even worse, such trend will possibly hinder the growth of national productivity and harm the balance between labor supply and demand. It is also not conducive to stably implementing the principle of distributing according to work performance or "more work, more pay".

In conclusion, from the short-term perspective, the excessive psychological pressure caused by overwork will not only cause the gradual reduction of employees' work efficiency and creativity, but also seriously affect their health, thus making their future development limited. Shepard, a scholar, came to a conclusion that a $10 \%$ increase in overtime results in a $2.4 \%$ decrease in productivity [1]. In the long run, since this over- extensive operation and insufficient humanistic management will result in the disharmony of labor relations, excessive overwork is fairly unfavorable to the long-term development of enterprises.

It turns out that Internet employees urgently need to seek an outlet for pressure and other negative emotions, so that their personal lives can get back on track, and self-growth and self-worth can be reasonably realized in appropriate work time.

\section{Research and analysis on ways of releasing psychological pressure of Internet employees}

Some Internet companies provide a variety of soft benefits such as leisure facilities in addition to resting space at the workplace, and unveil personalized work regulations, so as to relieve working stress via creating a more relaxing and comfortable work environment for employees. For instance, Bilibili, a Chinese videosharing company, pays great attention to the happy working mood of employees. It not only supports employees to bring pets to work, but also divides special space for employees to play with their pets. What's more, Bilibili also organizes a wide range of fun activities during festivals and invites their families to come, as well as creates various surprises for employees, allows them to adjust their emotions through entertainment for better work efficiency.

However, the limitations of such soft benefits mentioned above cannot substantially solve the psychological pressure of employees. For example, some team-building entertainment activities have gradually deviated from their core purpose due to the influence of bad workplace culture and etiquette. As a result, some team-building activities have become a spiritual or economic burden for employees. In addition, the introduction of some personalized regulations does not take into account the majority of employees' benefits. And, improper implementation will affect work efficiency in the least, but will cause more or less physical or psychological harm to other employees.

All in all, the distinctive soft benefits launched by Internet companies have gradually become part of their corporate culture. On the surface, they create a relaxing and pleasant working atmosphere that becomes an invisible booster in attracting more young people to join. However, in essence, such soft benefits have not really relieved the psychological pressure of employees. Under the unchangeable background of fast development and high work pressure, it still requires further exploration and practice that how to use existing technology and scientific pressure relief methods to help employees reduce their psychological pressure. 


\section{Exploration on other ways to relieve the pressure on Internet employees}

\subsection{Creating a healing and relaxing environment by offering space to empty themselves spiritually}

With the evolution of human civilization, privacy has become one of the basic requirements of human beings[2]. However, in Internet companies, the highintensity work and high-frequency personnel interactions make employees lack the time to be alone and self-talk. Compared with the high-frequency sensory stimulation by going to bright lights, the dialogues between people and themselves in quiet solitude help to restore self-spirit and reshape of self-worth. Currently, psychological researches on quiet solitude indicate that solitude is an inherent psychological phenomenon of individuals and is closely related to personal emotions. Anthony Storr, a British psychologist observes that the ability to be alone is manifested in individuals experiencing inner emotions, changing attitudes, and easing disappointment through contact with their inner world[3]. When individuals are alone, they can effectively detect emotional changes, regulate negative emotions, and enhance their positive energy. Therefore, Internet companies can create a healing space where individuals can be alone in the office leisure area, which is convenient for employees to talk to themselves, calm their minds, and empty themselves spiritually.

This requires the solitary space to be formed under certain scenes that can have a healing effect, thereby having a subtle influence on improving people's mental health and enhancing the sense of personal security. Furthermore, such a private space is conducive to creating a solitary environment for employees, helping to release stress and increase work happiness.

\subsection{Using environmental perception to provide employees with multi-sensory comfort}

Environmental perception is a process in which a sense organ acquires external information and extracts various features from external stimuli, and then allows the relations between perceived objects and background to participate in the formation of people's perceptions. Although this process is more complicated than a single sensory stimulus, it enables people to perceive the environmental evaluation based on environmental intuition under the stimulation of multiple sensory organs, and then integrates the environmental evaluation into forming the subsequent behavioral judgment and personal values. An experiment of "sensory deprivation", conducted by Baker Houston, a Canadian scientist, suggests that maintaining connection and balance between individuals and the environment is a necessary condition for a normal life of an individual, and the varied and rich sensory stimulation ensures this balance state . However, the long-term high-load work of Internet employees makes it difficult to have the opportunity to feel the surrounding environment. Consequently, there is also an imbalance of the connection between individuals and the environment. To change the imbalance, multisensory stimulation is an effective means.

Apart from the healing space, offering multi-sensory comfort stands another beneficial way to help Internet employees temporarily withdraw from busy work tasks and get physical relaxation, such as listening to music and watching green landscapes, etc. From a visual point of view, when designing office and leisure space, companies can choose warm-toned items and soft lighting to make employees feel warm and relaxed; from a tactile point of view, it is recommended to select simple logs, soft knits, pure cotton and linen, etc., since these materials have a sense of intimacy and calmness, which makes people feel humanistic care and warmth; from the auditory point of view, white noise, light music and other soothing music can be used to relax employees' nerve system.

In summary, if Internet companies create a private or personal leisure space where employees can receive perceptions of the external environment via multiple senses, employees will be physically and mentally relaxed in their leisure time. This will be a crucial way to solve the problem that Internet employees find it difficult to effectively release their psychological pressure. Also, it helps to create a more humanistic working environment and a more harmonious labor relationship.

\section{Design and Practice of the healing interactive device with healing function}

\subsection{Design concept and design demonstration}

With the rapid development of Chinese Internet companies, the employees have to work under huge pressure for long hours and find it difficult to relieve stress. In the long run, it is unfavorable sustain the development of both employees and the companies, nor is it conducive to forming the harmonious social labor relations. In response to this problem, the research designs an interactive device called Restart, through combining the healing design with arduino, an opensource development technology. Specifically, with balloons made of clouds, soft chairs and carpets, and headphones that play white noise, the device is dedicated to creating an immersive solitary relaxation scene for Internet employees, so that they can release pressure through the multiple physical senses such as seeing, hearing and touching. 


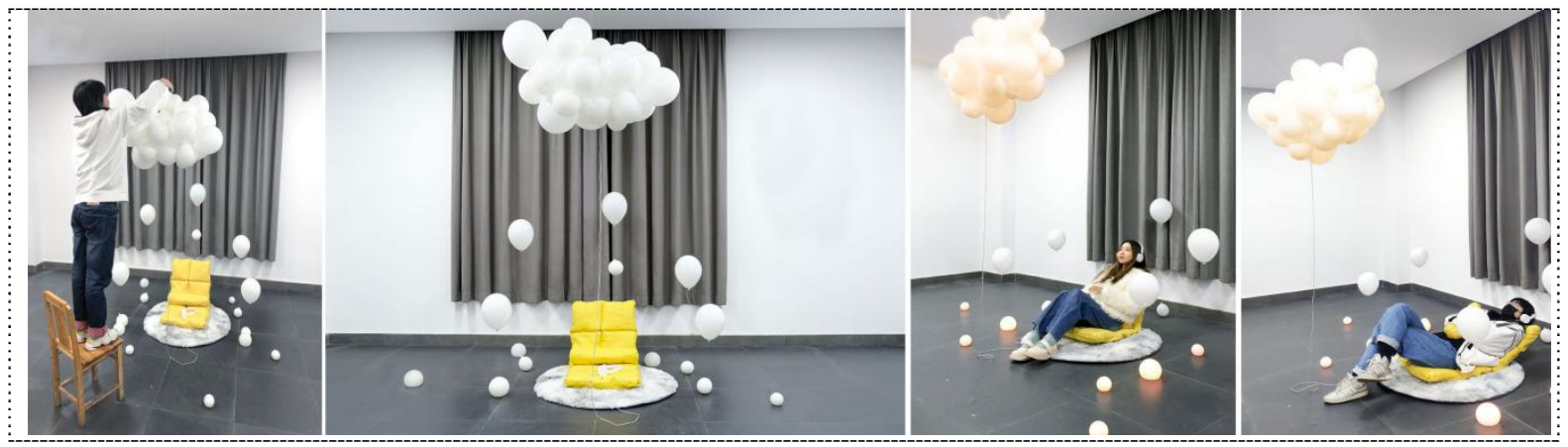

Figure 1. Design production and presentation

The entire device is composed of balloons, angleadjustable seats, earphones, carpets and LED lights. The cloud composed of white balloons complements the soft natural white noise in the earphones, as if the scene pulls users from high-intensity work into a vivid and quiet natural environment. The immersive experience can further stimulate the user's senses and assist them to obtain spiritual relaxation. The warm yellow soft cushions and white carpet can give users a gentle tactile feedback. What's more, the yellow and white device can enable users to have clean and bright visual enjoyment via creating a more effectively relaxing environment.

\subsection{Technical Analysis and Practice}

The interactive device utilizes arduino chips and various components to control the whole operation. It also applies pressure sensors, ultrasonic modules, and mp3 modules and LED lights, and interacts with users through programming. In detail, the pressure sensor is used to detect whether the user is sitting on the seat, and if so, then the whole device is triggered. The mp3 module and the LED lights control the sound and light respectively. The ultrasonic module behind the seat can sense the change of the chair at different angles and feed it back to the mp3 module and LED lights, in order to make corresponding changes for users.

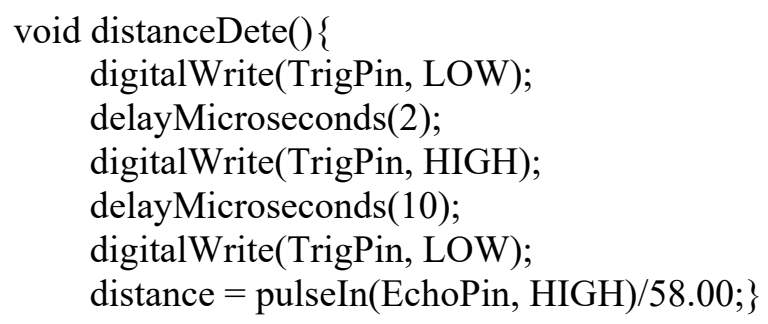

Figure 2. Code setting of the pressure sensors

The entire device experience is divided into three stages as below.

In the first stage, when the user sits on the seat in a relaxed mood, the pressure sensor senses the pressure and drives the LED lights and the mp3 module to work, thus triggering the device. Then, the user can enjoy the soft warm light radiated from the balloon clouds and the pleasant birdsong from the headphones, as if he or she was in the forest for an instant.

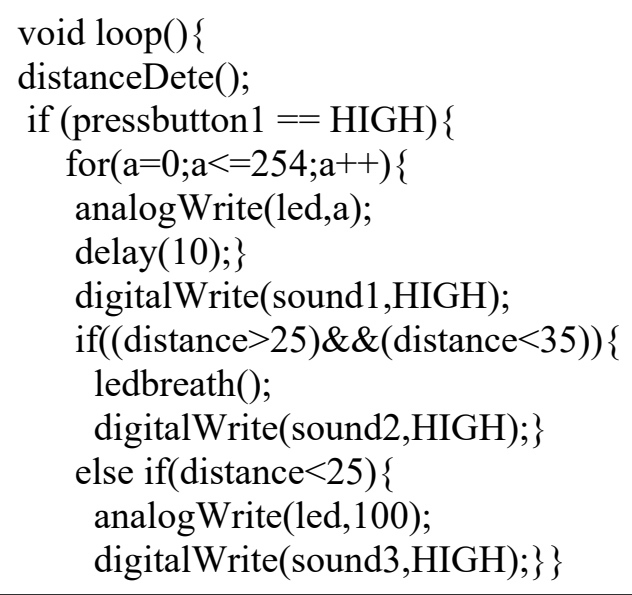

Figure 3. Code setting of the mp3 module 
In the second stage, users seek a more comfortable relaxed state. If users lean back, the white noise in the earphones will be converted into a softer sound of jungle wind than the first stage, along with reduced decibel and the lower brightness of cloud lights. Meanwhile, the lights turn from steady to softly flashing ones and such twinkling lights relax users at a deeper level.
In the third stage, if the user continues to lean back further, the white noise in the headset will turn into a clear raindrop sound, the lights return to be steady but with less brightness. Under the guidance of the light and sound, the user gradually enters deep rest. When the user gets up after a break, the device shuts off and the seat automatically returns to the original and static position.

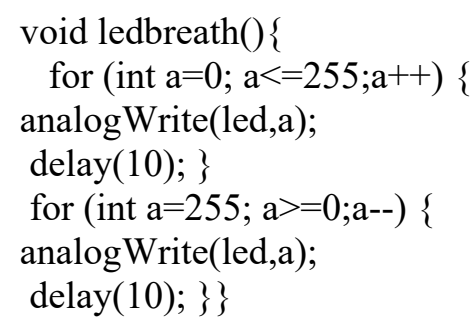

Figure 4. Code setting of the LED lights

The device combines open-source development technology with interactive art, focusing on the vivid and rich sensory experience, in order to allow Internet employees to experience the sound rest during tight work intervals, relieve their psychological pressure, and help them maintain high efficiency. The research also expects that this way of rest will set up a trend in the future, attracting more people to contribute to relevant research.

\section{Summary}

In comparison with the soft benefits to relieve pressure of Internet workers, this research has a more direct and effective influence on employees from a physiological perspective. Restart, the interactive device, integrates healing design with arduino open-source development technology to offer workers burdened with high-pressure an immersive experience combining vision and hearing. Restart aims to help employees release their psychological pressure, adjust their working state, and ease their fatigue; thereby, they can quickly restore energy, continue high work efficiency, and enhance creativity, thus forming a virtuous circle. Further, it can also improve the productivity of the companies and help to shape a friendly working atmosphere, putting forward new solutions for maintaining sound and harmonious social labor relations.

\section{References}

1. Shepard, E.\& T. Clifton, 2000, Are Longer Hours Reducing Productivity in Manufacturing?, International Journal of Manpower, 21(7) pp.540553.

2. Li Dan. Research on the Indoor Environment Design of Office Leisure Space Under the Healing Concept[D]. Hefei University of Technology, 2016.

3. Gao Shuang. The Effect of Solitary Satisfaction on Emotions[D].Harbin Normal University, 2014.
4. Lou Ruiping.\& Li Bei.\& Qi Xiaomei. Research on the Growth Path, Corporate Strategy and Management Strategy of Chinese Internet Companies [J/OL]. Management Critics:114[2021-01-10].

https://doi.org/10.14120/j.cnki.cn115057/f.20191127.002.

5. Xiao Feijun. Research on Overwork of employees in Chinese Internet Companies[D].Inner Mongolia Normal University, 2020. 